
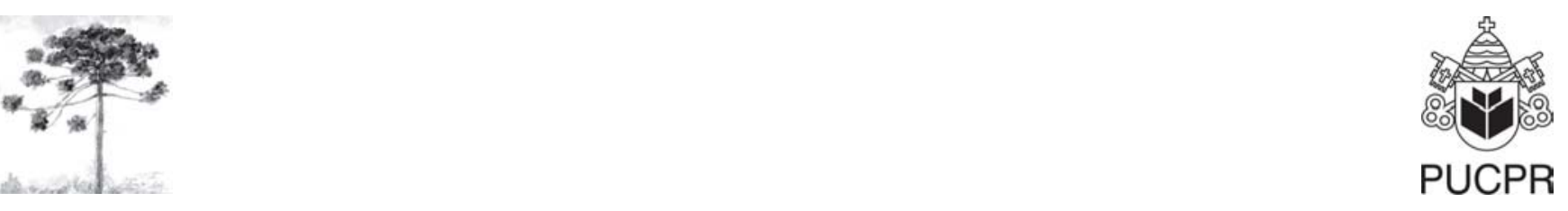

\title{
PARASITISMO POR Giardia sp., Cryptosporidium sp. E Cystoisospora sp. EM NUTRIA (Myocastor coypus) NO ESTADO DO RIO GRANDE DO SUL, BRASIL
}

\author{
Parasitism by Giardia sp., Cryptosporidium sp. and Cystoisospora sp. \\ in nutria (Myocastor coypus) in Rio Grande do Sul State, Brazil
}

\footnotetext{
Aleksandro Schafer da Silva ${ }^{1}$, Stéfano Leite $\mathrm{Dau}^{2}$, Luciana Faccio ${ }^{3}$, Régis Adriel Zanette ${ }^{4}$, Silvia Gonzalez Monteiro ${ }^{5}$

${ }^{1}$ Curso de Medicina Veterinária da Universidade Federal de Santa Maria (UFSM), Santa Maria, RS - Brasil, e-mail: aleksvet2002@Yahoo.com.br

${ }^{2}$ Curso de Medicina Veterinária da Universidade Federal de Santa Maria (UFSM), Santa Maria, RS - Brasil, e-mail: stedau@portoweb.com.br

${ }^{3}$ Curso de Medicina Veterinária da Universidade Federal de Santa Maria (UFSM), Santa Maria, RS - Brasil, e-mail: lucianabfaccio@hotmail.com

${ }^{4}$ Curso de Medicina Veterinária da Universidade Federal de Santa Maria (UFSM), Santa Maria, RS - Brasil, e-mail: regnitro@yahoo.com.br

${ }^{5}$ Departamento de Microbiologia e Parasitologia da UFSM, Santa Maria, RS - Brasil, e-mail: sgmonteiro@uol.com.br
}

\section{Resumo}

O objetivo deste trabalho foi registrar o parasitismo natural por Giardia sp., Cryptosporidium sp. e Cystoisospora sp. em nutria (Myocastor coypus) oriundas do município de Alegria, RS, Brasil. Foram analisadas amostras de fezes, pelo método de centrífugo-flutuação com sulfato de zinco, de sete roedores da espécie $M$. coypus capturados em seu ambiente natural e posteriormente soltos. Nas amostras analisadas, observou-se infecção por Giardia sp. (2 animais), infecção mista por Giardia sp. e Cryptosporidium sp. (2), infecção mista por Cryptosporidium sp. e Cystoisospora sp. (1) e infecção por Cryptosporidium sp. (1). Apesar do parasitismo, os animais não apresentavam sinais clínicos decorrentes disso.

Palavras-chave: Myocastor coypus; Protozoário; Animais silvestres. 


\begin{abstract}
The aim of this study was to report the natural parasitism by Giardia sp., Cryptosporidium sp. and Cystoisospora sp. in nutria (Myocastor coypus) from the municipality of Alegria, Rio Grande do Sul state, Brazil. Fecal samples of seven rodents of the specie M. coypus were analyzed by the centrifugal-flotation method with zinc sulphate. The animals were captured in their environment and released after the collection of the feces. Infection by Giardia sp. was found in two animals, by Giardia sp. and Cryptosporidium sp. in another two, by Cryptosporidium sp. and Cystoisospora sp. in one animal and by Cryptosporidium sp. in another animal. Although the parasitism the animals did not show any clinical signs due to the disease.
\end{abstract}

Keywords: Myocastor coypus; Protozoan; Wild animals.

Nutria (Myocastor coypus: Rodentia; Myocastoridae) é um roedor de natureza herbívora natural da região Sul da América Latina. Esse animal possui uma taxa de reprodução relativamente alta, uma tolerância a variações climáticas e hidrológicas, além de uma capacidade de adaptação e de dispersão elevada, o que faz com que sua espécie tenha ampla distribuição geográfica (1).

Conforme a literatura, em nutrias é relatado o parasitismo por helmintos das espécies Graphidioides myocastoris sp., Trichuris myocastoris, Dipetalonema sp., Hippocrepis myocastoris sp., e Rodentolepis sp. (2). A contaminação por protozoários gastrintestinais em vertebrados pode ocorrer por meio da ingestão de cistos ou oocistos esporulados, os quais podem estar presentes nas fezes, pastagens, água e nos alimentos $(3,4)$. A giardíase é uma infecção comum em animais vertebrados, sendo causada por um protozoário flagelado do gênero Giardia da família Hexamitidae, pertencente à ordem Diplomonadida (5). O Cryptosporidium sp. é um parasito obrigatório, intracelular, da família Cryptosporodidae, que se desenvolve por endodiogenia (4). Já o Cystoisospora sp. pertence à família Sarcocystidae da ordem Eucoccidiorina e a infecção dos hospedeiros ocorre pela ingestão de oocistos esporulados (6).

O potencial zoonótico de protozoários a partir de hospedeiro silvestre é ainda pouco estudado no Brasil, visto isto, o objetivo deste trabalho foi relatar o parasitismo natural de Giardia sp., Cryptosporidium sp. e Cystoisospora sp. em nutria (Myocastor coypus) no Rio Grande do Sul.
Foram analisadas amostras de fezes de sete nutrias da espécie $M$. coypus oriundos do município de Alegria, RS. Os roedores adultos, machos (4) e fêmeas (3), estavam em ambiente natural com área de mata e açude. Estes foram capturados com ajuda de gaiolas de tela $(70 \times 70 \times 50)$ iscadas com mandioca (Manihot esculenta), de onde foram coletadas fezes frescas, armazenadas em sacos plásticos e mantidas refrigeradas em caixa de isopor com gelo, até serem processadas no Laboratório de Parasitologia Veterinária da Universidade Federal de Santa Maria pelo método de centrífugo-flutuação com sulfato de zinco (técnica de Faust e cols, 1939) (7). Os animais capturados foram soltos no seu hábitat após a coleta das fezes. A avaliação da carga parasitária foi baseada na classificação de alguns autores (8) (infecção leve 1-100 oocistos, moderada 101-300 oocistos e elevada mais de 301 oocistos/lâmina). As fezes foram mantidas na estufa a $27^{\circ} \mathrm{C}$ por cinco dias para esporulação dos oocistos e posterior identificação (7).

Dos sete animais avaliados, dois apresentavam infecção por Giardia sp., dois continham infecção mista por Giardia sp. e Cryptosporidium sp., um dos roedores estava com infecção mista por Cryptosporidium sp. e Cystoisospora sp. e outro apresentava nas fezes somente infecção por oocistos de Cryptosporidium sp. Uma das amostras avaliadas apresentou-se negativa para protozoários, no entanto, todas as amostras avaliadas apresentavam ovos de Cestoda e Nematoda. Os gêneros Giardia e Cryptosporidium foram encontrados nos animais em infecções 
leve, moderada e elevada. Já o gênero Cystoisospora foi observado causando infecção leve no animal.

O protozoário do gênero Giardia, observado neste estudo, já foi relatado por outros autores (9) em Castor canadensis e Myocastor coypus em hábitat no Texas, onde observaram uma incidência de 30\% e $66,7 \%$ de positividade para o parasito, respectivamente. Nesse estudo, a incidência da giardiase em nutrias foi superior a $50 \%$ dos animais avaliados, semelhante ao trabalho relatado anteriormente (9). Conforme a literatura, o protozoário em questão pode causar infecção em seres humanos, cães, gatos, bovinos, suínos, ovinos e cavalos (10), levando a grandes perdas econômicas.

Nesse estudo, registra-se o primeiro caso de parasitismo por Cryptosporidium sp. em nutrias (Myocastor coypus). No entanto, este protozoário já foi relatado em outro trabalho realizado em Massachusetts, parasitando Castor canadensis, roedor da mesma ordem da nutria (11). A ocorrência da infecção por Cryptosporidium em pequenos mamíferos silvestres e roedores já foi relatada nas espécies Akodon serrensis, Oryzomys ratticeps, Mus domesticus, Apodemus sylvaticus e Clethrionomys glareolus $(12,13)$. Possivelmente este seja o primeiro registro de parasitismo devido à dificuldade de acesso a animais silvestres, o que dificulta pesquisas relacionadas ao parasitismo destes vertebrados.

A infecção leve em um dos roedores por Cystoisospora sp. trata-se do primeiro caso de parasitismo em $M$. coypus, protozoário este que causa comumente, em animais domésticos jovens, doença clínica (6), no entanto, neste estudo, o animal não apresentava diarréia, que é sinal clínico típico da enfermidade. Já em um estudo realizado com camundongos infectados experimentalmente com Cystoisospora obioensis, observou-se redução no ganho de peso dos animais infectados em comparação ao grupo controle (14), mostrando que o coccídeo em questão, apesar de não apresentar sinal clínico (diarréia), causa alterações no desenvolvimento dos animais parasitados.

Com base na análise das fezes, concluíse que Giardia sp., Cryptosporidium sp. e Cystoisospora sp. são parasitas de $M$. coypus em seu ambiente natural. Esses animais podem ser considerados reservatórios destes protozoários, pois não apresentavam sinais clínicos.

\section{REFERÊNCIAS}

1. Bolkoyic ML, Ramadori D. Manejo de Fauna Silvestre en la Argentina. Programas de uso sustentable. Buenos Aires: Dirección de Fauna Silvestre, Secretaría de Ambiente y Desarrollo Sustentable; 2006. 168 p.

2. Barbero BB, Cabello C, Kinoed JE. Helminto fauna de Chile. V. Nuevos parásitos del coipo, Myocastor coypus. Boletim Chileno de Parasitologia. 1979; 34:26-31.

3. Luwwig KM, Frei F, Alvares Filho F, Ribeiro-Paes JT. Correlation between sanitation condition and intestinal parasitosis in the population of Assis, State São Paulo. Revista da Sociedade Brasileira de Medicina Tropical. 2000;32(5):547-55.

4. Fayer R, Morgan U, Upton SJ. Epidemiology of Cryptosporidium: transmission, detection and identification. International Journal for Parasitology. 2000;30:12-13.

5. Olson ME. A Giardíase e o uso da vacinação para o controle da infecção. [S.1.: s.n]; 2000.

6. Urquhart GM, Armour J, Duncan JL, Dunn AM, Jennings FW. Parasitologia Veterinária. $2^{a}$ ed. Rio de Janeiro: Guanabara Koogan; 1998; 273 p.

7. Hoffman RP. Diagnóstico de parasitismo veterinário. Porto Alegre: Sulina; 1987. 156 p.

8. Pinto RM, Vicente JJ, Noronha D, Gonçalves L, Gomes DC. Helminth parasites of conventionally maintained laboratory mice. Memórias do Instituto Oswaldo Cruz. 1994;89(1):33-40.

9. Dunalp GD, Thies ML. Giardia sp. in Beaver (Castor canadensis) and Nutria (Myocastor coypus) form East Texas. The Journal of Parasitology. 2002;88(6):1254-1258.

10. Lallo MA, Rodrigues LCS, Bondan EF. Giardíase em cães e gatos. Clínica Veterinária. 2003;43:40-46. 
11. Fayer R, Santín M, Trout JM, DeStefano S, Koenen K, Kaur T. Prevalence of microsporidia, Cryptosporidium sp., and Giardia sp. in beaver (Castor Canadensis) in Massachusetts. The Journal of Zoo and Wildlife Medicine. 2006;37(4):492-497.

12. Dall'olio AJ, Franco RMB. Occurrence of Cryptosporidium spp. in small wildlife mammals from three mountainous areas in the Brazilian Southeastern region. Arquivo Brasileiro de Medicina Veterinária e Zootecnia. 2004;56(1):25-31.

13. Chalmers RM, Sturdee AP, Bull SA, Miller A, Wright SE. The prevalence of Cryptosporidium parvum and C. muris in Mus domesticus, Apodemus sylvaticus and Clethrionomys glareolus in an agricultural system. Parasitology Reseach. 1974;83:478-482.

14. Oliveira FCR, Lopes CWG, Massad FV, Melos PS. Influência da infecção por Cystoisospora obioensis no ganho de peso de camundongos albinos. Revista Brasileira de Parasitologia Veterinária. 2002;11(2):103-107.

Recebido: 14/05/2006

Received: 05/14/2006

Aceito: $24 / 11 / 2006$

Accepted: 11/24/2006 